\title{
Medienkompetenzvermittler: \\ Die Rolle von Medienunternehmen in der Schule
}

\author{
Elfi Heinke \& Michael Sengl
}

Abstract

Unter dem Einfluss der Digitalisierung steht der Journalismus in Deutschland vor großen Herausforderungen wie seiner Finanzierung, seiner Glaubwürdigkeit und »Fake News«-Vorwürfen. Eine gesteigerte Medienkompetenz in der Bevölkerung könnte einen Beitrag dazu leisten, diesen zu begegnen. In diesem Aufsatz werden die Ergebnisse einer explorativen, qualitativen Befragung von Medienunternehmen präsentiert, die einen Einblick geben, warum und auf welche Art und Weise diese Medienkompetenz insbesondere an Schulen vermitteln.

\section{Einleitung}

Jeden Tag stehen Medienschaffende aus Zeitung, Radiostationen oder Fernsehanstalten vor großen Herausforderungen: Wir recherchieren aufwändig, wir prüfen Aussagen, wir stehen für Glaubwürdigkeit und Transparenz. Gleichzeitig kämpfen wir gegen eine zunehmende Skepsis bei Lesern und Zuhörern durch politische Kampagnen und Fake News, die sich hartnäckig über das Netz verbreiten. [...] Vor allem junge Leser wollen wir ansprechen, sensibilisieren und nicht zuletzt auch aufklären. Denn Medienbildung ist Demokratiebildung. (Stadtmüller, Böhm \& Franke, 2019)

Mit diesen Worten laden die Stuttgarter Zeitung, die Stuttgarter Nachrichten und die Landeszentrale für politische Bildung Baden-Württemberg zum Schülermedientag 2019 in Baden-Württemberg ein. Damit sprechen sie eine große Herausforderung an, mit der der Journalismus aktuell konfrontiert ist. In einer Zeit, in der die Digitalisierung große Umbrüche in der Finanzierung, Produktion und Distribution von Medienprodukten mit sich bringt, in der sich die Gesellschaft zunehmend in verbitterte Gegner*innen und brennende Befürworter*innen traditioneller Medien spaltet, in der sogenannte alternative Nachrichtenquellen an Bedeutung und »Fake News« an Reichweite gewinnen, bietet die Vermittlung von Medienkompetenz, speziell Nachrichtenkompetenz, einen aussichtsreichen Ansatzpunkt. 
Medienkompetente Rezipient*innen sind in der Lage, eine aktive Entscheidung für Qualitätsjournalismus zu treffen und ihre Rolle als mündige Bürger*innen wahrzunehmen. Die folgende Untersuchung liefert einen ersten Einblick, was Medienunternehmen in Deutschland zur Vermittlung von Medienkompetenz beitragen und warum sie das tun.

\section{Medienkompetenz}

Medienkompetenz oder Media Literacy ist ein zentrales Konzept der Medienpädagogik. Als Folge der wachsenden Bedeutung von Internet und Multimedia wird der Begriff seit Anfang der 1990er Jahre verstärkt diskutiert und umfasst im wissenschaftlichen Diskurs vor allem »Wissensbestände über Medien sowie die Fähigkeit, Medien souverän bedienen, kritisch beurteilen und kreativ gestalten zu können« (Hugger, 2008, 93). Im deutschsprachigen Raum gilt Dieter Baacke als einer der Pionier*innen der Medienkompetenzforschung. Er differenziert in seiner Definition von Medienkompetenz die vier Teilbereiche Medienkritik, -kunde, -nutzung und -gestaltung. Medienkritik bezieht sich auf die Fähigkeit, durch den Medienwandel angestoßene gesellschaftliche Veränderungen zu analysieren und sowohl in Bezug auf sich selbst als auch die soziale Verantwortung zu reflektieren. Medienkunde meint das Wissen über das eigene Mediensystem sowie die Fähigkeit zur Handhabung neuer Geräte. Daran anknüpfend umfasst der Aspekt der Mediennutzung sowohl die passive als auch aktive Nutzung von Medienangeboten. Mediengestaltung als vierte Dimension beschreibt letztlich die Kompetenz, sowohl systemisch innovative als auch ästhetisch kreative Inhalte anzubieten (Baacke, 1996).

Für den Schulkontext konkretisiert Tulodziecki (1998) fünf zentrale Aspekte: 1) Auswahl und Nutzung von Medienangeboten unter Beachtung von Handlungsalternativen, 2) Eigenes Gestalten und Verbreiten von Medienbeiträgen, 3) Verstehen und Bewerten von Mediengestaltungen, 4) Erkennen und Aufarbeiten von Medieneinflüssen und 5) Durchschauen und Beurteilen von Bedingungen der Medienproduktion und -verbreitung.

Diese frühen Definitionsversuche finden sich auch im heute gültigen Kompetenzrahmen der Kultusministerkonferenz wieder. Dieser umfasst die sechs Kompetenzbereiche "Suchen, Verarbeiten und Aufbewahren«, »Kommunizieren und Kooperieren«, »Produzieren und Präsentieren«, »Schützen und sicher Agieren«, »Problemlösen und Handeln« und »Analysieren und Reflektieren« (Kultusministerkonferenz, 2016). Als essenziell 
erscheint in Zeiten von »Fake News « vor allem ein Aspekt der Medienkompetenz, die sogenannte Nachrichtenkompetenz.

\section{Nachrichtenkompetenz}

Gerade vor dem Hintergrund der Kompetenzvermittlung durch Medienunternehmen steht der Teilbereich der Medienkompetenz im Fokus dieses Beitrags, der sich mit Nachrichten beschäftigt. Die Nachrichtenkompetenz, auch News Media Literacy genannt, bezieht sich analog zur Media Literacy auf die einzelnen Teilbereiche der Nachrichtennutzung (Maksl, Craft, Ashley \& Miller, 2017). Dabei differenzieren beispielsweise Malik, Cortesi \& Gasser (2013) folgende Dimensionen: 1) ein Verständnis für die Rolle von Nachrichten in der Gesellschaft, 2) die Motivation, Nachrichten aktiv zu suchen, 3) die Fähigkeit, Nachrichten als solche zu erkennen, 4) diese kritisch zu bewerten und 5) diese eigenständig zu produzieren. Als Zielvorstellung für nachrichtenkompetente Rezipient*innen definieren sie dabei »empowered citizens « (Malik et al., 2013, 8), die in der Lage sind, sich am demokratischen Prozess zu beteiligen. Daraus ergibt sich auch der Sinn der Abgrenzung von Medien- und Nachrichtenkompetenz: Während Medienkompetenz als allgemeines Register von Fähigkeiten zur Nutzung massenmedial vermittelter Inhalte ein sehr umfassendes Konzept darstellt, bezieht sich die Nachrichtenkompetenz als spezieller Teilbereich auf die Fähigkeit zur kritischen und reflektierten Nutzung von journalistischen Inhalten und damit auf die Grundlage der Befähigung zur Teilnahme am demokratischen Prozess (Ashley, Maksl \& Craft, 2013; Maksl, Ashley \& Craft, 2015; Tully \& Vraga, 2018).

Damit stellen Nachrichtenkompetenz und insbesondere die kritische Mediennutzung einen wesentlichen Faktor im Umgang mit »Fake News« und Desinformation dar. In mehreren Studien zeigte sich außerdem, dass Nachrichtenkompetenz auch Auswirkungen auf andere Faktoren haben kann. Laut Craft, Ashley \& Maksl (2017) lässt sich ein Zusammenhang zwischen Nachrichtenkompetenz und der Skepsis bei der Mediennutzung (Maksl et al., 2015), dem Vertrauen in die Medien (Ashley, Poepsel \& Willis, 2010) sowie der Glaubwürdigkeitsbeurteilung (Carr, Barnidge, Lee \& Tsang, 2014) herstellen. Zudem wirkt sich ein höheres Level von Nachrichtenkompetenz positiv auf die Motivation aus, qualitativ hochwertige Nachrichten zu konsumieren (Maksl et al., 2015). Aus diesen Erkenntnissen lässt sich die hohe Relevanz der Nachrichtenkompetenz für den Journalismus herleiten, der sich aktuell mehreren Herausforderungen gleichzeitig stellen muss. 


\section{Situation des Journalismus}

Der öffentliche Diskurs über das Vertrauen in den Journalismus hat sich in den vergangenen Jahren insbesondere vor dem Hintergrund der Migrationsbewegungen ab 2015 intensiviert (Jackob et al., 2019). Aber auch wenn 2019 mit etwa 28 Prozent ein immer größerer Teil der Bevölkerung den etablierten Medien misstraut, so spricht ihnen auch ein immer größerer Teil, etwa 43 Prozent, explizit das Vertrauen aus - insgesamt steigt der Anteil derjenigen, die die plakativen Vorwürfe gegenüber einer sogenannten Lügenpresse zurückweisen, mit 58 Prozent auf den bisher höchsten im Rahmen der Mainzer Langzeitstudie Medienvertrauen gemessenen Wert an (Schemer et al., 2020). Demzufolge ist eine Polarisierung in der Gesamtbevölkerung zu erkennen, die in der Forschung auf eine ebenfalls immer stärker polarisierte Debatte über die Glaubwürdigkeit der Medien zurückgeführt wird (Jackob et al., 2019). Für den Journalismus bedeutet das, sich mit gut einem Viertel der Bevölkerung auseinandersetzen zu müssen, das zwar noch klassische Medien nutzt, allerdings signifikant häufiger auf alternative Medienangebote zurückgreift (Jackob, Schultz, Ziegele, Schemer \& Quiring, 2017). Die besagten Personen »finden sich überdurchschnittlich häufig am rechten Rand des politischen Spektrums. Sie sind formal niedriger gebildet, deutlich politikverdrossener und sie haben Angst, dass sich ihre wirtschaftliche Situation in der Zukunft verschlechtern wird « (Schemer et al., 2020).

Doch nicht nur diese Bevölkerungsgruppe stellt eine Belastung für die finanzielle Situation des Journalismus dar. Seit der Jahrtausendwende hat der Zeitungsjournalismus etwa 30 Prozent der Erlöse aus dem Kerngeschäft verloren (Lobigs, 2016). ${ }^{1}$ Gerade die jüngere Zielgruppe konsumiert Nachrichteninhalte hauptsächlich online. Die Online-Angebote klassischer Medienhäuser erwirtschaften bislang allerdings weder nennenswerte Werbe-, noch bedeutende Vertriebseinnahmen. Im Online-Werbebereich konkurriert der Journalismus heute mit mächtigen Intermediären wie Google oder Facebook und ist zusätzlich von einem massiven Preisverfall für einzelne Werbeanzeigen betroffen (Lobigs, 2016). Um Kosten zu sparen und eine Querfinanzierung der digitalen Angebote durch klassische Printprodukte zu

1 Die finanzielle Situation des privaten Rundfunks ist weitaus weniger ernst, dieser trägt allerdings auch weniger zur nachrichtlichen Berichterstattung bei (Lobigs, 2016). Der öffentlich-rechtliche Rundfunk ist aufgrund seiner Finanzierung aus dem Rundfunkbeitrag von der Finanzierungsdebatte - abgesehen von Diskussionen über die Höhe des Beitrags oder gar seiner kompletten Abschaffung - weitgehend ausgenommen. 
ermöglichen, sind auf dem Pressemarkt starke Konzentrations- und Konsolidierungstendenzen erkennbar. Medienunternehmen schließen sich zusammen, bauen Personal ab, bündeln Ressourcen und Redaktionen - die Auswirkungen auf die Qualität und Vielfalt der Berichterstattung sind dabei umstritten (Lobigs, 2016).

Sinkende Auflagenzahlen und der Einfluss der Digitalisierung auf Erlösund Distributionsmodelle stellen den Journalismus auch im Bereich der Produktion und Organisation vor neue Herausforderungen. So entwickelt sich die »medienvermittelte, öffentliche Kommunikation von einer sozial selektiven, einseitigen, linearen, zentralen und intransparenten zu einer partizipativen, interaktiven, netzartigen, dezentralen und transparenten Kommunikation « (Neuberger, 2017, 2-3). Dieser Wandel hat Auswirkungen auf mehreren Ebenen: Neben der bereits erläuterten Konkurrenz klassischer Medien und neuer Intermediäre um Werbekunden stellen diese eine wichtige symbiotische Plattform für die gezielte Ausspielung und Bewerbung journalistischer Inhalte dar. Doch nicht nur der Vertrieb, sondern auch die Recherche und Produktion von medialen Inhalten verändert sich durch die Digitalisierung. So ist die Nutzung von Suchmaschinen und sozialen Netzwerken zu einem Standardtool der journalistischen Arbeit geworden. Mit dem Datenjournalismus hat sich gar eine neue Disziplin herausgebildet (Neuberger, 2017). Beiträge werden crossmedial aufbereitet, die Grenzen einzelner Mediengattungen verschwimmen und auch die Redaktionsorganisation wandelt sich vielerorts von klassischen ressortgetrennten Strukturen hin zu sogenannten vollintegrierten Newsrooms mit zentraler Themensteuerung (García Avilés, Meier, Kaltenbrunner, Carvajal \& Kraus, 2009).

Nach Christoph Neuberger (2017) hat die Digitalisierung den Journalismus zusammenfassend in drei Krisen geführt: eine ökonomische Krise als Folge der Umbrüche auf dem Publikums- und Werbemarkt, eine Identitätskrise in Bezug auf die Abgrenzung neuer Online-Konkurrenten von klassischen journalistischen Medien und eine Qualitätskrise, angelehnt an die Polarisierung des Vertrauens in den Journalismus. ${ }^{2}$ In dieser angespannten Situation scheint eine Betrachtung der Initiativen von Medienunternehmen zur Vermittlung der zuvor beschriebenen äußerst relevanten Medienkompetenz vielversprechend.

2 Neuberger spricht hier von einer Krise, »die sich in einem Verlust an Glaubwürdigkeit niederschlagen soll« (Neuberger, 2017, 22). Neuere Studien wie Schemer et al. (2020) zeigen jedoch, dass es sich dabei vielmehr um eine gesellschaftliche Polarisierung als einen Vertrauensverlust handelt. 


\section{Medienkompetenzvermittlung durch Medienunternehmen}

Für Medienunternehmen scheint es vor dem Hintergrund dieser Herausforderungen eine logische Konsequenz zu sein, sich für eine Steigerung der Medienkompetenz der Rezipient*innen einzusetzen. Betrachtet man den Forschungsstand zur Medienkompetenzvermittlung durch Medienunternehmen, fällt das Fazit jedoch ernüchternd aus: Zum Zeitpunkt des Verfassens dieses Beitrags konnten die Autor*innen keine Studie im deutschsprachigen Raum finden, die sich explizit mit diesem Thema auseinandersetzt. Das ist umso verwunderlicher, da der im Rahmen dieser Arbeit ausdifferenzierte Medienkompetenzbegriff ein breites Spektrum von Fähigkeiten umfasst, die gerade im journalistischen Berufsalltag von zentraler Bedeutung sind - von der Mediennutzung für die Recherche, der kritischen Reflexion von Medieninhalten bis hin zur Produktion journalistischer Inhalte.

Diesen Zusammenhang greift auch Alexandra Durner in ihrer 2009 eingereichten Dissertationsschrift auf, in der sie ein journalistisches Unterrichtsprojekt zur politischen Medienbildung konzipiert. Konkret fasst sie dabei Grundzüge des journalistischen Arbeitens als Basiskompetenzen auf, die in der Schule vermittelt werden sollen. Dabei geht sie über den Einsatz als mediendidaktisches Instrument hinaus und betrachtet die kritische sowie analytische Auseinandersetzung mit journalistischen Darstellungen, beispielsweise in der Politikberichterstattung, als zentralen Zugang zur politischen Bildung (Durner, 2009). In ihrer Arbeit hebt sie neben einer Reihe anderer Initiativen das journalistische Projekt Zeitung in der Schule hervor, bei dem sich Schüler*innen unterstützt von Journalist*innen mit verschiedenen Aspekten der Mediennutzung und -produktion auseinandersetzen (Durner, 2009).

Abgesehen von dieser Arbeit finden journalistische Medienkompetenzprojekte kaum Erwähnung in wissenschaftlichen Arbeiten zur Vermittlung von Medienkompetenz. Umso wichtiger erscheint es, einen ersten Überblick über solche Projekte zu schaffen und damit die Grundlage für weitere Forschung zu legen.

\section{Forschungsfragen}

Aus diesem bisher sehr spärlichen Forschungsstand in Verbindung mit den theoretischen Vorüberlegungen ergeben sich für die vorliegende Studie folgende Forschungsfragen: 
- FF1: Wie sind Initiativen zur Vermittlung von Medienkompetenz seitens der Medienunternehmen in Deutschland ausgestaltet?

- FF2: Inwiefern fühlen sich Medienunternehmen in Deutschland für die Vermittlung von Medienkompetenz verantwortlich?

- FF3: Welche Motivation steht hinter den Initiativen?

Zur Beantwortung dieser Forschungsfragen wurden qualitative Leitfadeninterviews geführt, deren methodischer Hintergrund im Folgenden beschrieben wird.

\section{Methode}

Ziel dieser explorativen Studie war es, herauszufinden, wie und warum sich Medienunternehmen in Deutschland im Bereich der Vermittlung von Medienkompetenz engagieren. Ihre Ergebnisse sollen als Basis für weitere, quantitative Forschung auf diesem Gebiet dienen.

Da bei der Konzeption des Leitfadens nicht auf etablierte Konstrukte zurückgegriffen werden konnte, wurde der Leitfaden vollständig eigens konstruiert. Der Aufbau folgte dem Prinzip vom Allgemeinen zum Speziellen und gliedert sich in drei Teile: das Verständnis der jeweiligen Gesprächspartner*innen von Medienkompetenz, dem Verantwortungsgefühl sowie Chancen und Grenzen bezüglich der Medienkompetenzvermittlung durch Medienunternehmen, dem Aufbau und den Zielen des konkreten Projektes, seiner Zielgruppe und Entstehungsgeschichte.

Die Auswahl der Interviewpartner*innen basierte vor allem auf zwei Kriterien. Die Befragten mussten 1) einem journalistischen Medienunternehmen in Deutschland angehören und 2) in einer leitenden oder zumindest zentralen Position in dem Medienkompetenz-Projekt des Unternehmens engagiert sein.

Obwohl die kleine Stichprobe von sieben Expert*innen die deutsche Medienlandschaft nicht repräsentativ abbilden kann, wurde darauf geachtet, private und öffentlich-rechtliche sowie kleine und große Medienhäuser in die Studie aufzunehmen, die ein Medienkompetenz-Projekt anbieten.

Die Auswahl der Interviewteilnehmer*innen erfolgte auf Basis der Empfehlungen besonders engagierter Projekte seitens Thorsten Merkle von der Initiative Junge Leser, einem Netzwerk und einer Wissensdatenbank zum Thema Kinder- und Jugendengagement der Zeitungen. In Kombination mit 
einer Online-Recherche wurden sieben ${ }^{3}$ Expert*innen für die Untersuchung ausgewählt. Das Theoretical Sample bestand schließlich aus je zwei öffentlich-rechtlichen Rundfunkanstalten und überregionalen Tageszeitungen sowie drei Regionalzeitungen. Genauere Daten können der folgenden Tabelle entnommen werden.

\section{Tabelle 1: Interviewpartner*innen}

\begin{tabular}{|c|c|c|c|}
\hline $\begin{array}{l}\text { Medien- } \\
\text { gattung }\end{array}$ & Medienmarke & Projekt & Interviewpartner \\
\hline \multirow[t]{2}{*}{$\begin{array}{l}\text { überregio- } \\
\text { nale Tages- } \\
\text { zeitung }\end{array}$} & $\begin{array}{l}\text { Süddeutsche Zei- } \\
\text { tung (SZ) }\end{array}$ & $\begin{array}{l}\text { Schule \& Zei- } \\
\text { tung sowie die } \\
\text { SZ-Werkstatt- } \\
\text { gespräche }\end{array}$ & $\begin{array}{ll}\text { - } & \text { Mario Lauer, Gesamtvertriebs- } \\
\text { leiter der } S Z \\
\text { - Wilhelm Maassen, Geschäftsfüh- } \\
\text { rer des medienpädagogischen In- } \\
\text { stituts Promedia Maassen } \\
\text { - } \quad \text { Klaus Ott, Redakteur der } S Z \text { und } \\
\text { Mitorganisator der SZ-Werk- } \\
\text { stattgespräche }\end{array}$ \\
\hline & $\begin{array}{l}\text { Frankfurter All- } \\
\text { gemeine Zeitung } \\
\text { (FAZ) }\end{array}$ & Meine Zeitung & $\begin{array}{l}\text { Werner D'Inka, Mitherausgeber } \\
\text { der } F A Z \text { und Mitorganisator des } \\
\text { Projekts } \\
\text { Oliver Beddies, Bereichsleiter } \\
\text { Bildung der Stiftung Polytechni- } \\
\text { sche Gesellschaft Frankfurt am } \\
\text { Main (Mitorganisator des Pro- } \\
\text { jektes) }\end{array}$ \\
\hline \multirow[t]{2}{*}{$\begin{array}{l}\text { öffentlich- } \\
\text { rechtlicher } \\
\text { Rundfunk }\end{array}$} & $\begin{array}{l}\text { Bayerischer } \\
\text { Rundfunk (BR) }\end{array}$ & \multirow{2}{*}{$\begin{array}{l}\text { umfassendes } \\
\text { Angebot diver- } \\
\text { ser Projekte für } \\
\text { Schüler*innen } \\
\text { \& Lehrer*innen }\end{array}$} & $\begin{array}{l}\text { - Isabella Schmid, Leiterin der } \\
\text { Abteilung Medienkompetenzpro- } \\
\text { jekte des } B R\end{array}$ \\
\hline & $\begin{array}{l}\text { Südwestrundfunk } \\
\text { (SWR) }\end{array}$ & & $\begin{array}{l}\text { - Christine Poulet, Medienkompe- } \\
\text { tenzbeauftragte des } S W R\end{array}$ \\
\hline \multirow[t]{3}{*}{$\begin{array}{l}\text { regionale } \\
\text { Tageszei- } \\
\text { tung }\end{array}$} & $\begin{array}{l}\text { Mindener Tage- } \\
\text { blatt (MT) }\end{array}$ & MT clever & $\begin{array}{l}\text { - Nicola Waltemathe, Projektlei- } \\
\text { tung MT clever und stellvertre- } \\
\text { tende Marketingleitung } \\
\text { Lisa Meier, Projektteam MT cle- } \\
\text { ver } \\
\text { - Frank Sommer, Marketinglei- } \\
\text { tung }\end{array}$ \\
\hline & $\begin{array}{l}\text { Mittelbayerische } \\
\text { Zeitung (MZ) }\end{array}$ & $\begin{array}{l}\text { Klasse infor- } \\
\text { miert }\end{array}$ & $\begin{array}{l}\text { Dagmar Unrecht, Projektredak- } \\
\text { teurin für den Raum Regensburg }\end{array}$ \\
\hline & Main-Post (MP) & KLASSE! & $\begin{array}{l}\text { - } \begin{array}{l}\text { Peter Krones, Projektleitung } \\
\text { - } \\
\text { jekt }\end{array} \\
\text { jeke Faust, Redakteurin im Pro- }\end{array}$ \\
\hline
\end{tabular}

3 Ursprünglich wurden acht Medienunternehmen angefragt, die Madsack-Mediengruppe meldete sich nicht zurück. 
Alle Gespräche wurden im Zeitraum von Oktober 2019 bis Januar 2020 telefonisch geführt, anschließend transkribiert und einer qualitativen Inhaltsanalyse unterzogen. Durchschnittlich dauerte ein Interview circa 35 Minuten. Lediglich zwei Gespräche wichen mit einer Länge von knapp über 45 Minuten aufgrund ausführlicherer Antworten von den anderen ab.

\section{Ergebnisse}

Bevor die Forschungsfragen beantwortet werden, sollen zum besseren Verständnis der Ergebnisse zunächst die Zielgruppen der Projekte sowie die verschiedenen Definitionen des Begriffs Medienkompetenz seitens der Medienunternehmen vorgestellt werden.

\section{Zielgruppen der Projekte}

Alle sieben Medienunternehmen adressieren primär Schüler*innen sowie deren Lehrkräfte. Bis auf die Frankfurter Allgemeine Zeitung (FAZ), die Süddeutsche Zeitung (SZ) und das Mindener Tageblatt (MT) bieten die Medienhäuser ihre Projekte für alle Schularten und Altersstufen an. Das $M T$ widmet sich im Projekt $M T$ clever dagegen vor allem Kindergartenkindern sowie Grundschüler*innen, möchte aber perspektivisch mit den jungen Mediennutzer*innen mitwachsen. Die FAZ legt ihren Schwerpunkt im Projekt Meine Zeitung klar auf Schüler*innen der Jahrgangsstufen 6 bis 10, die $S Z$ auf die Mittel- und Oberstufe.

Neben dieser Hauptzielgruppe richten sich einige Medienunternehmen auch an ältere Menschen. ${ }^{4}$ Laut Christine Poulet (Südwestrundfunk, SWR) ist Medienkompetenz schließlich etwas, dass »zwingend erforderlich ist für alle Altersgruppen, da wir alle [Medien] konsumieren [...]. Es ist also ganz offensichtlich, dass die ganze Gesellschaft diese Medienkompetenz erwerben sollte« (Poulet, $S W R$ ). Jedoch ist das Angebot für diese Zielgruppe wesentlich geringer als für die Schulen, was sich auch in der Schätzung der

4 So unterstützt der SWR etwa das Projekt silver-tipps.de, das sich an Senior*innen wendet, und kooperiert mit Hochschulen und Bürgermedien. Der Bayerische Rundfunk $(B R)$ kooperiert unter anderem mit Volkshochschulen und lässt dort unter Anleitung Audioguides produzieren. Die $S Z$ veranstaltet sogenannte Werkstattgespräche an öffentlich zugänglichen Orten. Die Main-Post $(M P)$ und die Mittelbayerische Zeitung $(M Z)$ bieten über ihre jeweiligen Akademien kostenpflichtige Kurse an, etwa zur Smartphonenutzung. 
Medienkompetenzbeauftragten des $S W R$ zeigt, der zufolge sie ungefähr 70 Prozent ihrer Arbeitszeit Projekten für die Generation unter 25 Jahren widmet. Aus diesem Grund sowie dem klaren Fokus der Projekte auf Schüler*innen und ihre Lehrkräfte stehen für diese Untersuchung die Schulprojekte im Vordergrund der Auswertung.

\section{Definition Medienkompetenz}

Im Kontrast zu den sehr ähnlichen Zielgruppen der einzelnen Projekte unterscheiden sich die unterschiedlichen Verständnisse des Begriffs Medienkompetenz stärker voneinander. Die verschiedenen Definitionen decken ein breites Spektrum von relativ wenigen Fähigkeiten bis hin zu den klassischen vier Kompetenzen nach Baacke (1996) ab. Auf letztere bezogen sich die $S Z$ und der $S W R$ sehr deutlich und betonten dabei jeweils eine Fähigkeit besonders: Ein »eigenes Schreibtalent entwickeln« (Lauer, $S Z$ ), als ein Aspekt der Mediengestaltung, nimmt im Projekt der $S Z$ eine zentrale Rolle ein. Poulet (SWR) legt in ihrem Verständnis des Begriffs neben den Fähigkeiten nach Baacke vor allem Wert auf eine ethische Kompetenz, die ihrer Meinung nach darin »nicht ganz optimal abgedeckt ist « (Poulet, $S W R$ ). Sie ist neben Isabella Schmid $(B R)$ die Einzige, die neben Medienkompetenz auch gezielt von Nachrichtenkompetenz spricht, die »immer stärker im Mittelpunkt [steht] «(Poulet, $S W R$ ). Die Definitionen des Begriffs Medienkompetenz des $B R$, der $F A Z$ und der $M Z$ nähern sich ebenfalls stark dem Modell von Baacke (1996) an. Allerdings nennen diese Medienunternehmen in ihrer Definition hauptsächlich die Fähigkeiten Medienkritik, -kunde und -nutzung. Dennoch spielt, wenn auch nicht explizit in den Interviews erwähnt, die Mediengestaltung in ihren Projekten eine wichtige Rolle, da sie gezielt Medieninhalte produzieren lassen. Trotzdem betont Schmid $(B R)$, dass es »dabei jetzt weniger um die Technik, als vielmehr um die Inhalte [geht] Dagmar Unrecht ( $M Z$ ) hebt zudem die Fähigkeit zur Beurteilung von Quellen hervor: Die Schüler*innen sollen »ein Gefühl für Quellen [...] entwickeln, sozusagen [...] lernen, was seriöse, unabhängige Informationen sind und die [...] von subjektiven Einschätzungen [unterscheiden können] (Unrecht, $M Z$ ). Wie der Inhalt von Informationen zu bewerten ist und woher sie stammen, ist auch dem Projektteam des Mindener Tageblatt (Nicola Waltemathe, Lisa Meier, Frank Sommer) sehr wichtig. Damit fokussieren sie sich vor allem auf Medienkritik, -kunde und -nutzung. Mediengestaltung spielt eine deutlich untergeordnete Rolle, was klar auf die Zielgruppe des Projekts, Kindergartenkinder und Grundschüler*innen, zurückzuführen ist. 
Auch in der Definition von Medienkompetenz nach Peter Krones $(M P)$ sowie in seinem Projekt nimmt die Fähigkeit der Mediengestaltung keine tragende Rolle ein. Er betont dagegen ebenso wie Unrecht $(M Z)$ den regionalen Aspekt bezüglich der Fähigkeit zur Mediennutzung. Wie diese Regionalität auch in der Umsetzung der Projekte einen zentralen Platz einnimmt, zeigt sich im Folgenden.

\section{Umsetzung der Projekte}

Die Frage nach der Ausgestaltung der Medienkompetenzprojekte lässt sich auf zwei Ebenen beantworten. So gilt es zunächst die konzeptionelle Ebene $\mathrm{zu}$ betrachten, bevor die inhaltliche vorgestellt wird.

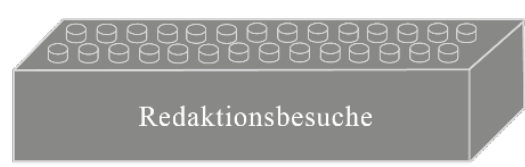

$+$

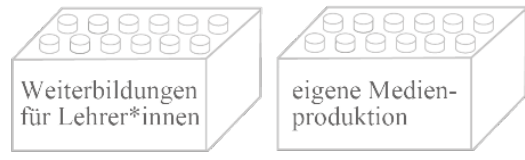

$+$

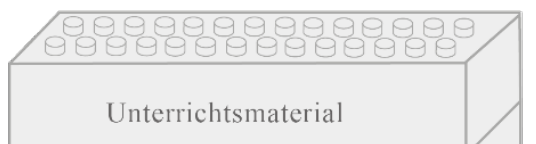

Besuche von Redakteur*innen

Bereitstellung des Mediums

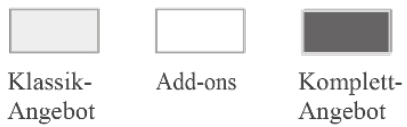

Abbildung 1: Inhalte der verschiedenen Angebote
Anhand der Aussagen der Interviewpartner*innen lässt sich die Gestaltung der Projekte in zwei Angebote sowie zwei Add-ons unterteilen. Den Inhalt der Angebote präsentiert die nebenstehende Grafik. Das Klassik-Angebot umfasst die Bereitstellung von Unterrichtsmaterial, Redakteur*innenbesuche in Schulen sowie die Bereitstellung eines Zugangs zum jeweiligen Medium und wird vom $M T$ umgesetzt. Die $S Z$ und die $M P$ ergänzen dieses Angebot um das Add-on eines Weiterbildungsangebots für Lehrer*innen, das unter anderem im Rahmen des Lehrermedientags der bayerischen Zeitungen umgesetzt wird. Zudem fügt die $S Z$ mit der eigenen Medienproduktion der Schüler*innen dem Klassik-Angebot ein zweites Add-on hinzu. Das Schreiben eigener Artikel sowie die Produktion audiovisueller Inhalte sind auch fester Bestandteil des Komplett-Angebots, das die $M Z$, der $B R$, der $S W R$ und die $F A Z$ anbieten. Hier wird das Klassik-Angebot um beide Add-ons und zusätzlich um Redaktionsbesuche ergänzt. Das heißt, es kommen nicht nur Journalist*innen in die Schulen, sondern es haben auch 
Schulklassen die Möglichkeit, die Redaktionen zu besuchen. Bei der $M Z$ können Schüler*innen etwa »zu uns ins Haus [kommen] und [...] mit unserem Social-Media-Experten zusammen eine Instagram-Story machen« (Unrecht, $M Z$ ). Dies verbindet den Redaktionsbesuch damit automatisch mit der Produktion eigener Medieninhalte seitens der Schüler*innen, wovon letzteres vor allem von der $F A Z$ und der $M Z$ besonders positiv bewertet wird. Diese positive Einstellung der Medienproduktion gegenüber teilen allerdings nicht alle Medienunternehmen. So kritisiert Krones $(M P)$, dass das eigene Schreiben von Artikeln nicht notwendig sei, um zu verstehen, wie man Medien richtig nutzt. Seiner Meinung nach ist »ein verpflichtendes Schreiben von Artikeln [...] Unsinn. Was Sie da bekommen, ist unlesbar. Das macht den Schülern keinen Spaß. Das macht den Lehrern keinen Spaß. Es macht den Redaktionen keinen Spaß.«

Eher einer Meinung sind die Medienhäuser, was die Weiterbildung von Lehrer*innen betrifft. Neben dem Lehrermedientag der bayerischen Zeitungen bietet vor allem der $B R$ in diesem Bereich viel an. So findet laut Schmid $(B R) »$ an jedem Schultag mindestens eine Lehrerfortbildung [statt] «. Zusätzlich gibt es eine Ausbildung zum beziehungsweise zur Medienexpert*in für Lehrkräfte, die sich über zwei Jahre erstreckt und mit einem vom Kultusministerium anerkannten Zertifikat abgeschlossen wird. Im Rahmen des Projektes Meine Zeitung der FAZ sowie der Stiftung Polytechnische Gesellschaft bereitet die Medienagentur mct Dortmund die Lehrkräfte pädagogisch in einem Workshop vor und begleitet sie während des Projekts.

Auch auf inhaltlicher Ebene ähneln sich die Projekte und zeigen damit einen erkennbaren Kanon, was Medienunternehmen den Schüler*innen und Lehrkräften vermitteln wollen. Hier stehen vor allem fünf Themen im Vordergrund. Jedes der sieben Medienhäuser möchte in seinem Projekt die Rolle und den Aufbau des eigenen Mediums erklären. Dabei dient die Zeitung beispielsweise im $S Z$-Projekt »als tagesaktuelles Lehrbuch« (Maassen, $S Z$ ), das »die persönliche Meinungsbildung und Interesse an Demokratie, Gesellschaft und Politik [fördert] « (Lauer, SZ). Mit dem zweiten zentralen Thema, den journalistischen Darstellungsformen, befassen sich auch alle Projekte. Laut Unrecht $(M Z)$ ist es heute schließlich »ganz wichtig für die Kinder und Jugendlichen [...], bewertende und kommentierende Aussagen [...] von sachlichen Texten unterscheiden zu lernen«. Auch im Projekt Schule \& Zeitung $(S Z) »$ setzen sich die Schülerinnen und Schüler mit Meinungsbildung und Meinungsmache [...] auseinander. Sie lernen, zwischen Information und Meinung zu unterscheiden und welche Grauzonen es hierbei gibt « (Maassen, $S Z$ ). Inhaltlich ebenfalls in diese Richtung geht es, dass außer beim $M T$ in allen anderen Projekten über die Arbeit von 
Journalist*innen gesprochen und versucht wird, diese nachvollziehbar zu machen. D'Inka $(F A Z)$ ist der Meinung, dass mehr Wissen über den Journalistenberuf nötig sei: »Und auch eine realistische Einschätzung dessen, was wir mit unserem Beruf [in der Gesellschaft] leisten können und wo vielleicht auch die Grenzen sind. « Klaus Ott (SZ) sagt: »Wir müssen erklären, wie wir arbeiten, wie wir recherchieren und redigieren, wie wir unsere Informationen prüfen, wie wir Nachrichten auswählen, wie wir entscheiden, was wie veröffentlicht wird, und vieles mehr. So entsteht Vertrauen. « Dabei kommt es laut Schmid $(B R)$ häufig auch zu einem Aha-Effekt, sowohl auf Seiten der Schüler*innen als auch der Lehrkräfte, und zu Aussagen wie »Wir hätten uns nie vorgestellt, dass da so ein Aufwand dahintersteht. Wir wissen gar nicht, woher sie eigentlich die Nachrichten bekommen«, was den Bedarf genau solcher Themen und Projekte noch einmal verdeutlicht.

Das wiederum zeigt auch die hohe Relevanz des fünften zentralen Themas, nämlich der Frage, was »Fake News« sind und wie man diese erkennen beziehungsweise verhindern kann. Bis auf das $M T$, dessen Projekt sich an Kindergartenkinder und Grundschüler*innen richtet, behandelt jedes der sechs Medienhäuser dieses Thema in ihrem Projekt. Die $M Z$ vermittelt in ihrem Projekt etwa, was seriöse und unabhängige Quellen sind. Die FAZ will laut D'Inka den Schüler*innen eine gesunde Skepsis mit auf den Weg geben: »Gebraucht euren eigenen Kopf, glaubt nicht alles. Versucht aber trotzdem, Quellen, die sich über längere Zeit als vertrauenswürdig herausgestellt haben, stärker zu nutzen und denen stärker zu trauen als anderen.« Sehr ähnlich ist auch die Aussage von Krones $(M P)$, in dessen Projekt an ein gewisses Misstrauen gegenüber Nachrichten unseriöser Medien appelliert wird. Um seriös von unseriös unterscheiden zu können, gebe es daher auch praktische Übungen im Lehrmaterial.

Zusätzlich zu diesen Hauptthemen widmen sich einzelne Projekte anderen aktuellen Fragestellungen. Der $B R$ und die $S Z$ haben etwa Informationen zu Extremismus und Hate Speech im Angebot, das $M T$, die $M P$ und die $M Z$ vergleichen verschiedene Medien miteinander. Diese Inhalte stellen im Überblick aller Projekte jedoch eher Randthemen dar. Nichtsdestotrotz betont das Projektteam von MT clever (MT), dass es wichtig sei, die Projekte stets aktuell zu halten und weiterzuentwickeln, um »tagtäglich im Fokus unserer Zielgruppe zu bleiben « und damit eine hohe Teilnahme an dem Projekt garantieren zu können. Letztlich bleibt die Frage, weswegen sich Medienunternehmen nun verantwortlich fühlen, Medienkompetenz zu vermitteln. 


\section{Verantwortungsgefühl}

Inwiefern sich Medienunternehmen für die Vermittlung von Medienkompetenz verantwortlich fühlen, lässt sich vor allem anhand zwei Arten von Verantwortung erklären: der Rollenverantwortung als Medienunternehmen beziehungsweise als Journalist*in und der Aufgabenverantwortung, die Medien als sogenannte vierte Macht in der Demokratie innehaben. Letztere spielt vor allem für die zwei öffentlich-rechtlichen Rundfunkanstalten $B R$ und $S W R$ eine wichtige Rolle, da sie sich qua Gesetz zur Erfüllung des Bildungsauftrages verantwortlich fühlen, der im Rundfunkstaatsvertrag (RStV) festgehalten ist. Poulet $(S W R)$ verweist außerdem auf ein Urteil des Bundesverfassungsgerichts $(2018)^{5}$, »wo noch einmal dezidiert beschrieben wird, dass sowohl der öffentlich-rechtliche als auch der private Rundfunk verpflichtet sind, den Auftrag zu übernehmen, Medienkompetenz in die Gesellschaft zu bringen« (Poulet, $S W R$ ). Schmid (BR) ergänzt, dass dies auch seitens des Publikums gefordert werde. So ist es laut der ARD-AkzeptanzStudie aus dem Jahr 2018 rund drei Viertel der deutschen Bevölkerung wichtig, dass sich die $A R D$ im Bereich Medienkompetenz engagiert (ARD, 2019). Was das Verantwortungsgefühl aus der gesellschaftlichen Aufgabe der Medien, die Förderung der Teilhabe am politischen Diskurs, betrifft, so wird diese ebenfalls vom $B R$ sowie der $S Z$ angesprochen. Letztere sieht ihre Aufgabe »in Zeiten von Influencern, Fake News und Verschwörungstheoretikern [...] darin, dazu beizutragen, die Weichen zu stellen für die Zukunft der Demokratie. Nur wer weiß, wie und wo man sich seriös und fundiert informiert, kann mitreden « (Maassen, $S Z$ ). Jedoch begründet die $S Z$ ihre Verantwortung zusätzlich auch mit zwei Fakten, die sich aus der Rollenverantwortung heraus ergeben. Auf diese beziehen sich vor allem die privatwirtschaftlichen Medienunternehmen, die nicht an den RStV gebunden sind. Hier zeigen sich primär drei Verantwortungsbereiche. Zum ersten ergibt sich die Verantwortung der einzelnen Journalist*innen daraus, das eigene Wissen in ihrer informierenden und erklärenden Rolle weiterzugeben. »Wer könnte diese Kompetenzen besser vermitteln als die Medienunternehmen, die tagtäglich damit umgehen?« (Lauer, SZ). Zum zweiten müssten Medienhäuser Transparenz bezüglich ihrer Arbeit schaffen und

5 Dabei bezieht sie sich auf die Leitsätze zum Urteil des Ersten Senats vom 18. Juli 2018, in dem das BVerfG urteilt: »Auch Projekte zur Förderung der Medienkompetenz [...] dienen der Veranstaltung von öffentlich-rechtlichem Rundfunk, jedenfalls soweit sie einen Bezug zum Mediennutzer aufweisen und dessen Kenntnisse des Mediums sowie den Umgang mit ihm verbessern sollen« (Bundesverfassungsgericht, 2018). 
damit Medienkompetenz fördern: »[Wir haben] viel, viel stärker die Aufgabe $[\ldots]$ als früher, zu erklären, was wir tun und warum wir das tun und von welchen Prinzipien wir uns leiten lassen. Also ganz grundsätzlich nicht nur in diesen Schulprojekten « (D'Inka, FAZ). Und zum dritten die Verantwortung, die sich aus der Art des Mediums ergibt, das sich verantwortlich fühlt, Medienkompetenz zu vermitteln. So begründen die $M Z$ und das $M T$ ihre Verantwortung aus ihrer Rolle als vertrauenswürdiges Medium heraus, das diesen Beitrag zur Medienbildung leisten muss, die $S Z$ aus ihrer Rolle als Leit- und Qualitätsmedium und die $M P$ sowie das $M T$ aus ihrer Rolle als regionale Tageszeitungen. Letztere erklären dies vor allem mit der hohen Reichweite in der jeweiligen Region sowie der Nähe zu ihren Nutzer*innen. »Wir sind am nächsten dran an den Menschen«, sagt das Projektteam des $M T$. »Wir sind diejenigen, die Medienkompetenz am besten vermitteln können, $[\ldots]$ weil wir hier direkt vor Ort die Lehrer kennen, die Rektoren und natürlich auch die Leser«, fügt Krones $(M P)$ hinzu. Jedoch ist das Verantwortungsgefühl eine Sache und die Motivation, die hinter den Projekten steht, eine andere.

\section{Motivation der Projekte}

»In erster Linie [dient das Projekt] dem Bildungsauftrag und soll nicht nur einen werblichen Aspekt haben«, sagt Lisa Meier vom MT. Unrecht (MZ) erklärt: »Natürlich sind wir ein Wirtschaftsunternehmen. Natürlich hoffen wir, dass wir mit diesem Projekt Kinder und Jugendliche überhaupt noch Heranführen an unser Angebot.« Bereits diese beiden Zitate verdeutlichen, dass hinter den Medienkompetenzprojekten nicht allein altruistische Motive stecken. Dennoch beschränkt sich die Motivation keinesfalls ausschließlich auf extrinsische Motive, auch intrinsische Gründe lassen sich für das Engagement finden. Diese ähneln den Verantwortungsgefühlen, die die Medienunternehmen bereits genannt haben. Sowohl die Tageszeitungen $M Z, F A Z, S Z$ als auch die öffentlich-rechtlichen Rundfunkanstalten $B R$ und $S W R$ vermitteln Medienkompetenz, weil sie Qualitätsjournalismus erklären und damit auch Transparenz schaffen wollen. Bis auf den $S W R$ sprechen die anderen Medienhäuser auch gezielt davon, Bürger*innen durch die Vermittlung von Medienkompetenz die »Teilhabe an einem politischen gesellschaftlichen Diskurs« (Schmid, BR) zu ermöglichen. Ein drittes intrinsisches Motiv nennen die $M P$ und die $S Z$. Nämlich, dass sie sich auch engagieren, weil sie Lehrer*innen zu guten Wissensvermittler*innen im Bereich Medienkompetenz weiterbilden möchten. Um sehr viele und nicht nur 
einzelne wenige Klassen erreichen zu können, »brauchen wir immer den Lehrer als Wissensvermittler" (Krones, $M P$ ). Schließlich setzten die verfügbaren Ressourcen auch Grenzen, weshalb etwa die $M P$ ihre Redakteur*innen nicht täglich in die Schulen schicken könne.

Dennoch sind diese intrinsischen Motive häufig auch verbunden mit extrinsischen, wie die Aussage von Unrecht (MZ) zeigt: »Es geht darum, Verständnis zu entwickeln auf Schülerseite [...] für die Arbeit, die wir als Regionalzeitung leisten [...]. Über dieses grundsätzliche Interesse an der regionalen Berichterstattung hoffen wir natürlich schon langfristig auch, Bindungen entstehen zu lassen. «Das heißt, neue Leser*innen zu gewinnen, was auch die $M P$ und das $M T$ als ein Motiv nennen. Zwar sei auch klar, dass das nie »eins zu eins« (Unrecht, $M Z$ ) funktionieren würde, aber Hoffnung darauf bestehe trotzdem. D'Inka $(F A Z)$ ist da pessimistischer und sagt, dass man sich keine Illusionen machen solle, dass bei diesem Projekt alle Schüler*innen zu Abonnent*innen würden. Er spricht daher eher von einem »gattungsökonomischen Interesse, dass bei jungen Leuten das Bewusstsein überhaupt wachgehalten wird, dass es noch etwas gibt, das heißt Zeitung«. Das Bewusstsein für dieses Medium wollen auch die $S Z$ und die $M Z$ schaffen. Damit einher gehen auch die Präsentation und Positionierung der eigenen Marke, die das $M T$ und die $F A Z$ ansprechen. Ein weiteres relevantes extrinsisches Motiv durch positive Verstärkung ist es, Vertrauen zu erhalten, neu zu schaffen oder wieder zu erlangen. Das ist der MP und dem SWR ebenso wichtig wie der $F A Z$. Schließlich bedeute weniger Vertrauen auch automatisch weniger Leser*innen (D'Inka, $F A Z$ ).

Ein letztes extrinsisches Motiv durch positive Verstärkung ist die Förderung der Lesekompetenz und -freude, die von der SZ, der FAZ und dem MT genannt wurde. Natürlich ließe sich hier auch argumentieren, dass dieses Motiv aus eigenem Antrieb heraus ein Grund der Medienhäuser sein könnte, Medienkompetenz zu vermitteln, wie es auch das Motiv der Förderung einer Bürgerkompetenz ist. Dennoch lässt sich nicht leugnen, dass die Fähigkeit, lesen zu können, ganz klar auch die Voraussetzung dafür ist, eine Tageszeitung rezipieren zu können, sodass hier eventuell mehrere Motive zusammenspielen.

Der Vollständigkeit halber muss auch die extrinsische Motivation durch negative Verstärkung genannt werden. Diese beschränkt sich allerdings allein auf den oben bereits genannten gesetzlich vorgeschriebenen Bildungsauftrag, den der $S W R$ und der $B R$ erfüllen müssen.

Dass die Motivation der Medienhäuser aber deutlich über extrinsische Motive hinausgeht, zeigt zudem die Tatsache, dass für alle Interviewpartner*innen die Projekte ihrer direkten, aber auch indirekten Kon- 
kurrenten auf dem Medienmarkt keinerlei Konkurrenz füreinander darstellen. »Ich sehe uns da eher insgesamt als Branche gefordert. [...] Es geht grundsätzlich darum, einfach den Wert der Medien und die Bedeutung im Rahmen des Projekts zu vermitteln. [...] Und da sind wir alle in einem Boot«, sagt Unrecht ( $M Z)$. Zudem besteht laut Schmid $(B R)$ schließlich eine sehr hohe Nachfrage nach solchen Initiativen. Hilfreich sei es daher auch, sich mit anderen Projekten auszutauschen $(F A Z, M T)$, da jeder von jedem etwas lernen könne (MT). Ähnlich sieht dies auch die $S Z$, die über den Austausch hinaus mehr oder weniger zur Zusammenarbeit auf diesem Gebiet aufruft. Ihr Motto lautet: »Gemeinsam erreichen wir mit großen Aktionen zum Beispiel rund um den Tag der Pressefreiheit am 3. Mai mehr, als wenn jeder für sich allein arbeitet«, wie Ott (SZ) sagt. So ließe sich etwa mit Landesmedienanstalten oder öffentlichen Trägern zusammenarbeiten. Zusätzlich könnten Tandems mit Universitäten und Kommunikationswissenschaftler*innen entstehen, die selbstständig über Journalismus sprechen, aber auch wissenschaftliche Hintergründe liefern und mithilfe von Forschung derartige Projekte evaluieren und eventuell verbessern können (SZ). Letzteres sollte ganz klar als Appell an Forschende in Deutschland verstanden werden, sich in solchen Projekten zu engagieren und auf dem Gebiet der Medienbildung nicht nur in universitären Kontexten aktiv zu werden oder wie D’Inka (FAZ) sagt: »Je mehr mitmachen, umso besser.«

\section{Diskussion}

Zusammenfassend lassen sich die Forschungsfragen dieser Untersuchung wie folgt beantworten: In den Interviews zeigt sich, dass sich die Initiativen zur Vermittlung von Medienkompetenz primär an Schüler*innen und deren Lehrkräfte wenden. Dabei reicht das Angebot von der Bereitstellung von Unterrichtsmaterial, kostenlosen Zeitungslieferungen und Redakteur*innenbesuchen bis hin zu Weiterbildungen für Lehrkräfte, Redaktionsbesuchen und einer eigenen Medienproduktion der Schüler*innen. Inhaltlich vermitteln die Projekte hauptsächlich Informationen zum jeweiligen Medium sowie der journalistischen Arbeitsweise, zur Unterscheidung zwischen meinungs- und faktenzentrierten Darstellungsformen und zum Thema dieses Sammelbandes: »Fake News«. Das Verantwortungsgefühl der einzelnen Medienhäuser ergibt sich primär aus ihrer Rollenverantwortung als Medienunternehmen, etwa das eigene Wissen weiterzugeben oder aber Transparenz bezüglich ihrer Arbeit zu schaffen, sowie ihrer Aufgabenverantwortung als vierte Macht in der Demokratie, etwa der Erfüllung des 
im RStV festgehaltenen Bildungsauftrags oder der gesellschaftlichen Aufgabe zur Förderung der Teilhabe am politischen Diskurs. Die Motive hinter den Initiativen sind weder ausschließlich extrinsisch und damit etwa mit dem Gewinn neuer Zielgruppen zu erklären, noch allein intrinsisch, zum Beispiel in der Vermittlung von Bürgerkompetenz begründet. Vielmehr ist es ein Zusammenspiel aus beiden Motivarten, die sich auch nicht immer getrennt voneinander betrachten lassen. Zwar gestaltet sich die organisatorische Umsetzung der Projekte teilweise schwierig, was hauptsächlich mit nicht ausreichenden Ressourcen sowie der teils fehlenden Motivation der Lehrkräfte, ihre Klassen für derartige Projekte anzumelden, zu erklären ist. Dennoch sehen die Medienunternehmen auch viele Chancen in ihren Initiativen. Mit dem Gewinn von Vertrauen oder der Eindämmung des Phänomens »Fake News« seien an dieser Stelle nur zwei genannt. Nicht zuletzt zeigt auch die Tatsache, dass keines der Medienunternehmen andere Projekte als Konkurrenz, sondern eher als Bereicherung für die jeweiligen Zielgruppen und die Gesellschaft sieht, wie wichtig ihnen diese Projekte sind.

Auch wenn diese Ergebnisse einen spannenden Einblick in dieses noch wenig erforschte Feld bieten, so können sie dennoch nur eine erste Momentaufnahme liefern. Eine Limitation der Studie ist ihr explorativer und qualitativer Charakter, der keine Rückschlüsse auf die Grundgesamtheit von Medienkompetenzprojekten deutscher Medienunternehmen erlaubt. Hinzu kommt, dass die Ergebnisse der Studie lediglich auf den Auskünften der Interviewpartner*innen beruhen und es gerade bei Themen wie Verantwortungsgefühlen und Motiven hinter Handlungen zu Effekten sozialer Erwünschtheit kommen kann. Daher sind Anschlussstudien unbedingt zu empfehlen. So würden sich etwa quantitative Inhaltsanalysen der Projektwebseiten und Unterrichtsmaterialien sowie quantitative Befragungen der Projektverantwortlichen anbieten, um einen Überblick zu allen derartigen Projekten deutschlandweit zu erhalten. ${ }^{6}$ Auch interessant wäre die Frage, wie Medienunternehmen diese Projekte finanzieren. Trotz dieser Limitationen bietet die Studie einen ersten Einblick in das Engagement deutscher Medien, der Gesellschaft Medienkompetenz zu vermitteln und damit auch dem Phänomen »Fake News« etwas entgegenzusetzen.

6 Einen guten Startpunkt hierfür bietet die im Nachgang dieser Studie gestartete Plattform Journalismus macht Schule (https://journalismus-macht-schule.org), die die Angebote einer ganzen Reihe von Medien und Bildungsorganisationen sammelt (Sadrozinski, 2020). 


\section{Literatur}

ARD (2019). Medienkompetenz zum Anfassen. Das war der ARD-Jugendmedientag 2019. Abgerufen von http://www.ard.de/home/die-ard/presse-kontakt/ pressearchiv/Medienkompetenz_Das_war_der_ARD_Jugendmedientag_2019/ 5813966/index.html.

Ashley, Seth; Maksl, Adam \& Craft, Stephanie (2013). Developing a News Media Literacy Scale. Journalism \& Mass Communication Educator, 68(1), 7-21. DOI: $10.1177 / 1077695812469802$.

Ashley, Seth; Peopsel, Mark \& Willis, Erin (2010). Media Literacy and News Credibility. Does Knowledge of Media Ownership Increase Skepticism in News Consumers? Journal of Media Literacy Education, 2(1), 37-46.

Baacke, Dieter (1996). Medienkompetenz - Begrifflichkeit und sozialer Wandel. In: Rein, A. von (Hrsg.), Medienkompetenz als Schlüsselbegriff (112-124). KlinkhardtVerlag.

Bos, Wilfried; Eickelmann, Birgit; Gerick, Julia; Goldhammer, Frank; Schaumberg, Heike; Schwippert, Knut; Senkbeil, Martin; Schulz-Zander, Renate \& Wendt, Heike (Hrsg.) (2014). ICILS 2013. Computer- und informationsbezogene Kompetenzen von Schülerinnen und Schülern in der 8. Jahrgangsstufe im internationalen Vergleich. Waxmann.

Bundesverfassungsgericht (2018). Leitsätze zum Urteil des Ersten Senats vom 18. Juli 2018. Abgerufen von https://www.bundesverfassungsgericht.de/SharedDocs/ Entscheidungen/DE/2018/07/rs20180718_1bvr167516.html.

Carr, D. Jasun; Barnidge, Matthew; Lee, Byung Gu \& Tsang, Stephanie Jean (2014). Cynics and Skeptics. Evaluating the Credibility of Mainstream and Citizen Journalism. Journalism \& Mass Communication Quarterly, 91(3), 452-470. DOI: 10.1177/ 1077699014538828.

Carretero, Stephanie; Vuorikari, Riina; Punie, Yves; European Commission \& Joint Research Centre (2017). DigComp 2.1. The Digital Competence Framework for Citizens. With Eight Proficiency Levels and Examples of Use. DOI: 10.2760/38842.

Craft, Stephanie; Ashley, Seth \& Maksl, Adam (2017). News Media Literacy and Conspiracy Theory Endorsement. Communication and the Public, 2(4), 388-401. DOI: $10.1177 / 2057047 \mathrm{t} 17725539$.

Durner, Alexandra (2009). Ein journalistisches Unterrichtsprojekt als politische Medienbildung. Eine qualitative Untersuchung von Medienbildung in einem journalistischen Unterrichtsprojekt in Haupt- und Realschulen. Universität Kassel.

García Avilés, José A.; Meier, Klaus; Kaltenbrunner, Andy; Carvajal, Miguel \& Kraus, Daniela (2009). Newsroom Integration in Austria, Spain and Germany. Models of Media Convergence. Journalism Practice, 3(3), 285-303. DOI: 10.1080/ 17512780902798638.

Hugger, Kai-Uwe (2008). Medienkompetenz. In: Sander, U.; F. von Gross \& K.-U. Hugger (Hrsg.), Handbuch Medienpädagogik (93-99). VS, Verlag für Sozialwissenschaften. 
Jackob, Nikolaus; Schultz, Tanjev; Jakobs, Ilka; Quiring, Oliver \& Schemer, Christian (2019). Medienvertrauen im Zeitalter der Polarisierung. Mainzer Langzeitstudie Medienvertrauen 2018. Media Perspektiven, 5, 210-220.

Jackob, Nikolaus; Schultz, Tanjev; Ziegele, Marc; Schemer, Christian \& Quiring, Oliver (2017). Medienzyniker und Medienfans. Merkmale eines gespaltenen Publikums. In: Haller, M. (Hrsg.), Öffentliches Vertrauen in der Mediengesellschaft (118-138). Herbert von Halem Verlag.

Kirchhoff, Susanne \& Prandner, Dimitri (2017). Die Paywall-Debatte Erkenntnisse aus internationalen Erfahrungen mit bezahlten Onlineinhalten. In: Aichberger, I.; G. Götzenbrucker; S. Kirchhoff; D. Prandner \& R. Renger (Hrsg.), Was bleibt vom Wandel? (179-200). Nomos Verlagsgesellschaft. DOI: 10.5771/ 9783845274539-178.

Kultusministerkonferenz (2016). Strategie der Kultusministerkonferenz »Bildung in der digitalen Welt«. Abgerufen von https://www.kmk.org/fileadmin/Dateien/ veroeffentlichungen_beschluesse/2018/Strategie_Bildung_in_der_digitalen_Welt_ idF._vom_07.12.2017.pdf.

Länderkonferenz MedienBildung (2008). Kompetenzorientiertes Konzept für die schulische Medienbildung. Abgerufen von https://lkm.lernnetz.de/files/Dateien_lkm/Dokumente/LKM-Positionspapier_2015.pdf.

Lobigs, Frank (2016). Finanzierung des Journalismus - von langsamen und schnellen Disruptionen. In: Meier, K. \& C. Neuberger (Hrsg.), Journalismusforschung (69138). Nomos Verlagsgesellschaft. DOI: 10.5771/ 9783845271422-69.

Maksl, Adam; Ashley, Seth \& Craft, Stephanie (2015). Measuring News Media Literacy. Journal of Media Literacy Education, 6(3), 29-45.

Maksl, Adam; Craft, Stephanie; Ashley, Seth \& Miller, Dean (2017). The Usefulness of a News Media Literacy Measure in Evaluating a News Literacy Curriculum. Journalism \& Mass Communication Educator, 72(2), 228-241. DOI: 10.1177/ 1077695816651970.

Malik, Momin M.; Cortesi, Sandra \& Gasser, Urs (2013). The Challenges of Defining »News Literacy«. SSRN Electronic Journal. DOI: 10.2139/ssrn.2342313.

Neuberger, Christoph (2017). Journalismus und Digitalisierung: Profession, Partizipation und Algorithmen [Expertise für die Eidgenössische Medienkommission EMEK für die »Kommunikationsordnung Schweiz: Perspektiven des Journalismus«]. Abgerufen von https://www.emek.admin.ch/inhalte/Journalismus_und_Digitalisierung Expertise_Neuberger.pdf.

Newman, Nic; Fletcher, Richard; Kalogeropoulos, Antonis; Levy, David A. L. \& Nielsen, Rasmus Kleis (2018). Reuters Institute Digital News Report 2018.

Primack, Brian A.; Gold, Melanie A.; Switzer, Galen E.; Hobbs, Renee; Land, Stephanie R. \& Fine, Michael J. (2006). Development and Validation of a Smoking Media Literacy Scale for Adolescents. Archives of Pediatrics \& Adolescent Medicine, 160(4), 369. DOI: 10.1001/archpedi.160.4.369.

Sadrozinski, Jörg (2020). Journalismus macht Schule. Abgerufen von https://journalis mus-macht-schule.org. 
Schemer, Christian; Jackob, Nikolaus; Quiring, Oliver; Schultz, Tanjev; Ziegele, Marc \& Jakobs, Ilka (2020). Langzeitstudie Medienvertrauen. Forschungsergebnisse der Welle 2019. Abgerufen von https://medienvertrauen.uni-mainz.de/forschungs ergebnisse-der-welle-2019/.

Senkbeil, Martin; Goldhammer, Frank; Bos, Wilfried; Eickelmann, Birgit; Schwippert, Knut \& Gerick, Julia (2014). Das Konstrukt der computer- und informationsbezogenen Kompetenzen in ICILS 2013. In: Bos, W.; B. Eickelmann; J. Gerick; F. Goldhammer; H. Schaumberg; K. Schwippert; M. Senkbeil; R. Schulz-Zander \& H. Wendt (Hrsg.), ICILS 2013. Computer- und informationsbezogene Kompetenzen von Schülerinnen und Schülern in der 8. Jahrgangsstufe im internationalen Vergleich (83-112). Waxmann.

Stadtmüller, Carola; Böhm, Wenke \& Franke, Thomas (2019). Fakten gegen Fake News! Erster Schülermedientag in der Region Stuttgart.

Tully, Melissa \& Vraga, Emily K. (2018). Who Experiences Growth in News Media Literacy and Why Does It Matter? Examining Education, Individual Differences, and Democratic Outcomes. Journalism \& Mass Communication Educator, 73(2), 167181. DOI: $10.1177 / 1077695817706572$.

Tulodziecki, Gerhard (1998). Entwicklung von Medienkompetenz als Erziehungs- und Bildungsaufgabe. Pädagogische Rundschau, 52(6), 693-709.

Vraga, Emily K.; Tully, Melissa; Kotcher, John E.; Smithson, Anne-Bennett \& Broeckelman-Post, Melissa (2015). A Multi-Dimensional Approach to Measuring News Media Literacy. Journal of Media Literacy Education, 7(3), 41-53. 